\title{
Theory of Impedance Loaded Loop Antennas and Nanorings From RF to Optical Wavelengths
}

\author{
Arnold F. McKinley
}

\begin{abstract}
The analytical theory of perfectly conducting thinwire closed-loop antennas with multiple loads in the periphery was formally derived in the 1950s and 1960s. In this paper, it is extended to loop antennas and nanorings for use in communications, in the "Internet of things," and as metamaterials. The new derivation relies on recent work from 2013 that incorporates the surface impedance of metal wires into the standard theory, thus pushing its applicability into the gigahertz, terahertz, and optical regimes. Surface impedance effects cause losses and phase shifts in the current within the loop, which in turn cause wavelength scaling and degradation of signal strength. These effects are modeled using a critical point transition model of permittivity and of the index of refraction. The new results therefore extend standard loop antenna theory so that it now includes characteristics of multiply loaded loops over a very broad spectrum from radio frequencies to the optical region. The new model is verified using modern simulation tools. The examples given here include resistive and capacitive loading.
\end{abstract}

Index Terms - Antenna theory, critical point model, Internet of things, loop antennas, metamaterials, multiply loaded antennas, nanorings, nanotechnology, wavelength scaling.

\section{INTRODUCTION}

$\mathbf{S}$ TANDARD loop antenna theory appeared in the 1950s and 1960s with the work in Storer [1] and $\mathrm{Wu}$ [2]. Solutions of the differential equations were difficult, even with the assumption of closed ring vanishingly thin perfectly conducting (PEC) wires. Later, lizuka [3] broke the need for a closed ring, enabling the theory to include multiple loads evenly spaced around the periphery. It was not until 2013 [4] that the surface impedance effects, which cause loss and phase shift, were incorporated into the closed-loop theory, using a critical point transition model of permittivity and of the index of refraction. This enhancement brought the standard closed ring theory to the gigahertz $(\mathrm{GHz})$, terahertz $(\mathrm{THz})$, and optical regions.

In this paper, the surface impedance effects are coupled with the multiply loaded ring theory so that the entire theory becomes available to researchers working in $\mathrm{GHz}$ and $\mathrm{THz}$ communication and in $\mathrm{GHz}, \mathrm{THz}$, and optical metamaterials.

In Section II, the original cumbersome matrix notation used to derive the theory of multiply loaded loops is replaced with

Manuscript received July 20, 2016; revised February 11, 2017; accepted March 8, 2017.

The author is with the Department of Electronic and Electrical Engineering, University College London, London WC1E 7JE, U.K. (e-mail: arni.mckinley@ucl.ac.uk).

Color versions of one or more of the figures in this paper are available online at http://ieeexplore.iee.org.

Digital Object Identifier 10.1109/TAP.2017.2682219

the easier Einstein notation. From the very first step, the derivation includes the characteristics of metals at high frequencies. The resulting new theory yields proper wavelength scaling, an important characterization of metals operating at very high frequency [5, Sec. 9.17] [6]. Two examples are given, one with resistive loads and one with capacitive loads. Conclusions follow with the Appendix giving a heretofore unreported derivation of the complete resistance, inductance, and capacitance of loop antennas, which, when new theory is included, yields these values for the entire spectrum, RF through optical. The second part of the Appendix gives proper simulation settings for verification of the new theory using CST's Microwave Studio (MWS) ${ }^{1}$ tool.

Two variables are important to understand. The first is the parameter $k_{b}=2 \pi b / \lambda$, where $\lambda$ is the incident or driving wavelength. Frequency increases as $k_{b}$ increases. The second is the thickness measure $\Omega=2 \ln (2 \pi b / a)$. Larger values of $\Omega$ refer to larger aspect ratios, $b / a$, and therefore to thinner wires. Loop antennas and nanorings where $\Omega \geq 10$ are said to follow the "thin-wire" approximation, for which all of the derivations here apply.

\section{Modern Derivation of Multiply LoAded Loops}

\section{A. Current Distribution}

The principle theory of closed thin-wire loop antennas, given in [1] and [2], was extended to $\mathrm{GHz}, \mathrm{THz}$, and optical frequencies by [7] and [4]. In summary, a delta-function voltage generator, $V_{0} \delta(\phi)$, across an infinitesimal gap at $\phi=0$ provides a broad spectrum source for the loop (see Fig. 1). The current distribution for a completely closed ring without load is given by

$$
\begin{aligned}
I(\phi) & =V_{0} \sum_{m=-\infty}^{\infty}\left[\frac{e^{i m \phi}}{Z_{m}^{\prime}}\right] \\
\text { where } Z_{M}^{\prime} & =j \pi \xi_{0} a_{m}+(b / a) Z_{s} .
\end{aligned}
$$

The standard theory uses only the single term $Z_{m}=$ $j \pi \xi_{0} a_{m}$. The addition of $(b / a) Z_{s}$ extends the theory to the higher frequency ranges. $Z_{s}$ is the surface impedance of the metal material of the wire in all frequency regimes. A detailed definition and discussion of this impedance is given in [4].

The input impedance $Z_{\mathrm{CL}}$ and the input admittance $Y_{\mathrm{CL}}$ of the closed loop are given by placing the driving input voltage

\footnotetext{
${ }^{1}$ Computer Simulation Technology AG, MWS. 2012. Darmstadt, Germany. www.cst.com.
} 


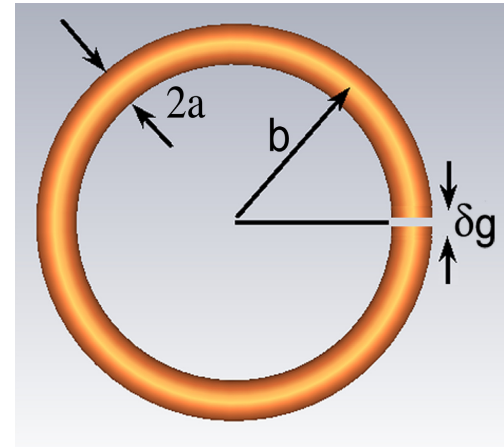

Fig. 1. Loop geometry. The gap is infinitesimally small and across it is placed a delta-function voltage generator to provide a broad spectrum source.

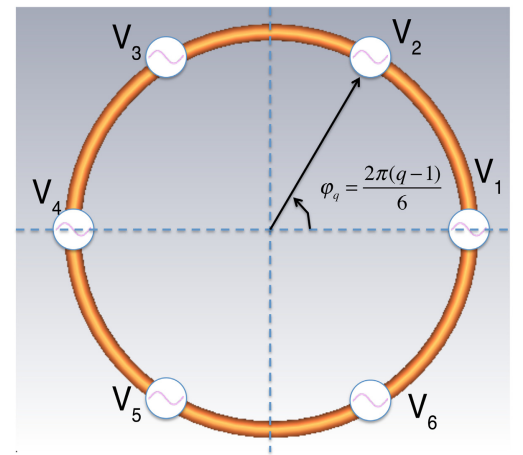

Fig. 2. Loop geometry of multiple sources and loads. The voltage sources marked " $V_{q}$ " are evenly spaced around the ring. The notation is short for " $V_{q} \delta\left(\phi-\phi_{q}\right)$ ".

at $\phi=0$

$$
Z_{\mathrm{CL}}=\frac{V_{0}}{I(0)}=\left[\sum_{m=-\infty}^{\infty} \frac{1}{Z_{m}^{\prime}}\right]^{-1}=\frac{1}{Y_{\mathrm{CL}}} .
$$

Iizuka [3] extended the low-frequency theory to include loads placed in the periphery of the loop. Unfortunately, the notation used in his paper is cumbersome and the more useful Einstein notation clarifies his approach and makes calculations easier. The derivation for loops with load impedances begins by first placing a number of $V_{q} \delta(\phi)$ voltage sources evenly around the ring separated by an angle $\Delta \phi=2 \pi / M$ where $M$ is the number of impedances, as shown in Fig. 2. Suppose $M=6$; the sources are then at the angles $\phi_{q}=2 \pi(q-$ $1) / 6, q=\{1, \ldots, 6\}$. The current in the ring results from a superposition of the currents due to all of the voltage sources, $V_{q}$. Each current has the form given by (1) and therefore is a distributed function of the angle, $\phi$. The total current is

$$
\begin{aligned}
I(\phi) & =\sum_{q=1}^{M} I_{q}(\phi) \\
& =\sum_{q=1}^{M}\left(\sum_{m=-\infty}^{\infty} \frac{e^{i m(\phi-2 \pi(q-1) / M)}}{Z_{m}^{\prime}}\right) V_{q} \\
& \equiv \sum_{q=1}^{M} Y(\phi, q) V_{q} .
\end{aligned}
$$

Since each $Z_{m}^{\prime}$ is given by (1), the surface impedance of ${ }_{101}$ the wire is automatically included and Iizuka's theory is automatically extended to the higher frequency range.

Note that at the source $p$

$$
\begin{aligned}
Y\left(\phi_{p}, q\right) & =\sum_{m=-\infty}^{\infty} \frac{e^{i m(2 \pi(p-q) / M)}}{Z_{m}^{\prime}} \\
& \equiv\left[Y_{p q}\right] .
\end{aligned}
$$

This lends itself to matrix notation. The term $\left[Y_{p q}\right]$ is a square matrix with $M \times M$ elements; $p$ is the row counter and $q$ the column counter. $Y(\phi, q)$ in (3) becomes a vector, $Y_{q}(\phi)$. Then (3) becomes

$$
\begin{aligned}
I(\phi) & =\sum_{q=1}^{M}\left[Y_{q}(\phi)\right]\left[V_{q}\right] \\
& =Y^{q}(\phi) V_{q}
\end{aligned}
$$

where the last line uses the Einstein summation rule (repeated indices are summed over); the upper index refers to the elements in a horizontal vector and the subscript counter refers to the elements in a vertical vector. Similarly

$$
\left[Y_{p q}\right] \rightarrow Y_{q}^{p} .
$$

In order to include impedances in the formalism, series impedances (voltage sinks) are added to the voltage sources. $V_{q}$ transforms as

$$
V_{q} \rightarrow V_{q}-Z_{q}^{k} I_{k} .
$$

Setting $V_{q}$ to 0 eliminates the generator leaving only the impedance and vice versa. The current $I_{k}$ is the current at the source (with its distinct counter to avoid confusion), that is, $I_{k}$ means $I\left(\phi_{k}\right)$. $Z_{q}^{k}$ is a square $M \times M$ diagonal matrix in which the diagonal terms are the impedances at each of the voltage sources. This approach provides enough information to find the current at each impedance node. Setting $\phi_{p}$ in (5) and using (6)

$$
\begin{aligned}
I_{p} & =Y_{p}^{q} V_{q} \rightarrow Y_{p}^{q}\left(V_{q}-Z_{q}^{k} I_{k}\right) \\
& =Y_{p}^{q} V_{q}-Y_{p}^{q} Z_{q}^{k} I_{k} \\
I_{p}+Y_{p}^{q} Z_{q}^{k} I_{k} & =Y_{p}^{q} V_{q} \\
\left(\mathcal{I}_{p}^{k}+Y_{p}^{q} Z_{q}^{k}\right) I_{k} & =Y_{p}^{q} V_{q} .
\end{aligned}
$$

The term $\mathcal{I}_{p}^{k}$ is the diagonal identity matrix. To simplify the notation, define

$$
f_{p}^{k} \equiv \mathcal{I}_{p}^{k}+Y_{p}^{q} Z_{q}^{k} .
$$

Equation (8) becomes

$$
I_{k}=\left[f^{-1}\right]_{k}^{p} Y_{p}^{q} V_{q}
$$

where the inverse of the matrix $f_{p}^{k}$ has been taken. When (7) and (10) are substituted into the sum (5), the current at any angle in the ring results

$$
\begin{aligned}
I(\phi) & =Y^{q}(\phi)\left(V_{q}-Z_{q}^{k} I_{k}\right) \\
& =Y^{q}(\phi) V_{q}-Y^{q}(\phi) Z_{q}^{k} I_{k} \\
& =Y^{j}(\phi) V_{j}-Y^{q}(\phi) Z_{q}^{k}\left[f^{-1}\right]_{k}^{p} Y_{p}^{j} V_{j} .
\end{aligned}
$$




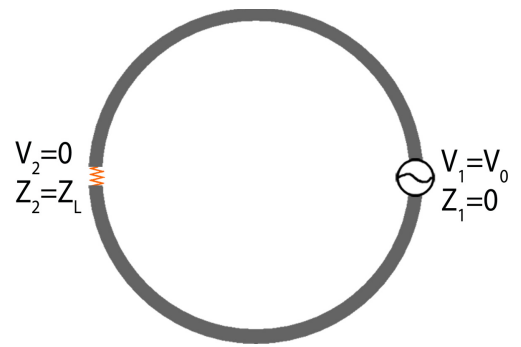

Fig. 3. Ring with one driving source and a load at $\phi=\pi$ to show a formal solution using (11).

The last line requires a change in the counter notation to prevent counter confusion. Notice the difference between (5) and (11). Each voltage generator $V_{q}$ in the former has been extended to include a load impedance.

\section{B. Input Impedance}

The driving point impedance and admittance occur when there is only one driving source. In this case, the source can be at any of the $\mathrm{M}$ angles spread evenly around the ring. Taking it at $\phi=0$, the impedance and admittance are calculated by dividing the source voltage, $V_{1}=V$, by the current at $\phi=0$

$$
Z_{\text {in }} \equiv \frac{V}{I(0)}=\left[Y^{1}(0)-Y^{q}(0) Z_{q}^{k}\left[f^{-1}\right]_{k}^{p} Y_{p}^{1}\right]^{-1} \text {. }
$$

\section{A. Resistive Loads}

As examples, let us explore singly loaded resistive and capacitive loops. In the first case, place in a PEC loop, a load impedance at $\phi_{2}=\pi$, and the drive source at $\phi_{1}=0$, as in Fig. 3. Then, $M=2, V_{1}=V$ volt, $V_{2}=0 ; Z_{2}^{2} \equiv Z_{L}$, while all other impedance elements are zero. The matrix elements, $Y_{p}^{q}$, are calculated as follows:

$$
Y=\left[\begin{array}{cc}
Y_{\mathrm{CL}} & Y_{\pi} \\
Y_{\pi} & Y_{\mathrm{CL}}
\end{array}\right]
$$

where

$$
Y_{\mathrm{CL}} \equiv \sum_{m=-\infty}^{\infty} \frac{1}{Z_{m}^{\prime}} \text { and } Y_{\pi} \equiv \sum_{m=-\infty}^{\infty} \frac{(-1)^{m}}{Z_{m}^{\prime}} .
$$

The matrix elements for $f$ are

$$
f=\left[\begin{array}{cc}
1 & Y_{\pi} Z_{L} \\
0 & 1+Y_{\mathrm{CL}} Z_{L}
\end{array}\right]
$$

and the current in (10) is

$$
\begin{aligned}
& I_{1}=\left[\frac{Y_{\mathrm{CL}}+Z_{L}\left(Y_{\mathrm{CL}} Y_{\mathrm{CL}}-Y_{\pi} Y_{\pi}\right)}{1+Y_{\mathrm{CL}} Z_{L}}\right] V \\
& I_{2}=\left[\frac{Y_{\pi}}{1+Y_{\mathrm{CL}} Z_{L}}\right] V .
\end{aligned}
$$

The total current is given by expanding (11)

$$
\left.I(\phi)=V\left[Y^{1}(\phi)-\frac{Y_{\pi} Z_{L}}{1+Y_{\mathrm{CL}} Z_{L}} Y^{2}(\phi)\right)\right] .
$$

These results reproduce Iizuka's equations (18), (19), and (22). The input impedance for the example can be found from (16)

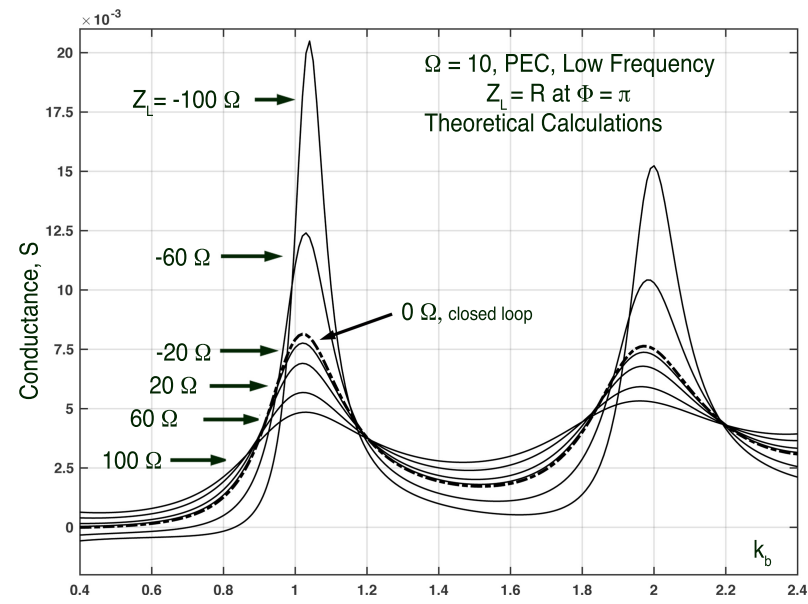

Fig. 4. Input admittance of an $\Omega=10$ and $2 \pi b=30$-m PEC loop with various resistive loads at $\phi=\pi$ using (18).

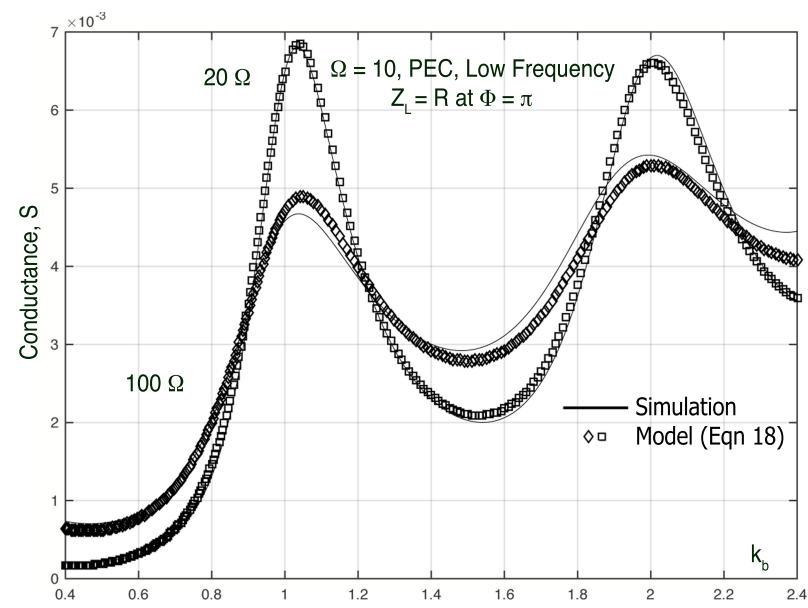

Fig. 5. 20- and 100- $\Omega$ curves of Fig. 4 compared with those given by simulation. See notes in the Appendix for simulation settings.

since $I_{1} \equiv I(0)$, or from (17) setting $\phi=0$

$$
Z_{\text {in }}=\frac{V}{I(0)}=\frac{1+Y_{\mathrm{CL}} Z_{L}}{Y_{\mathrm{CL}}+Z_{L}\left(Y_{\mathrm{CL}} Y_{\mathrm{CL}}-Y_{\pi} Y_{\pi}\right)} .
$$

The conductance of six different loop antennas where $Z_{L}=R=\{-100,-60,-20,20,60,100\}$, shown in Fig. 4 duplicates [3, Fig. 8]. See [3] for other resistive examples. The negative resistances were fabricated in [3] using Esaki diodes. Note that negative resistance can generate high resonances. Fig. 5 checks the validity of the model by comparing the 20 - and $100-\Omega$ curves of Fig. 4 with curves obtained by modern simulation. The correspondence is strong.

\section{B. Capacitive Loads}

As another example of the application of (18), set $Z_{L}$ to a capacitive reactive load. Using $\omega=k_{b} c / b$, where $c$ is the speed of light, the load may be expanded

$$
Z_{L}=Z_{\beta}=-j \frac{1}{\omega C}=-j \frac{\xi_{0}}{k_{b} l_{\epsilon}} .
$$

$k_{b}$ normalizes the reactance to the size of the loop and $l_{\epsilon}$ becomes a measure of capacitance. For example, a capacitor of 


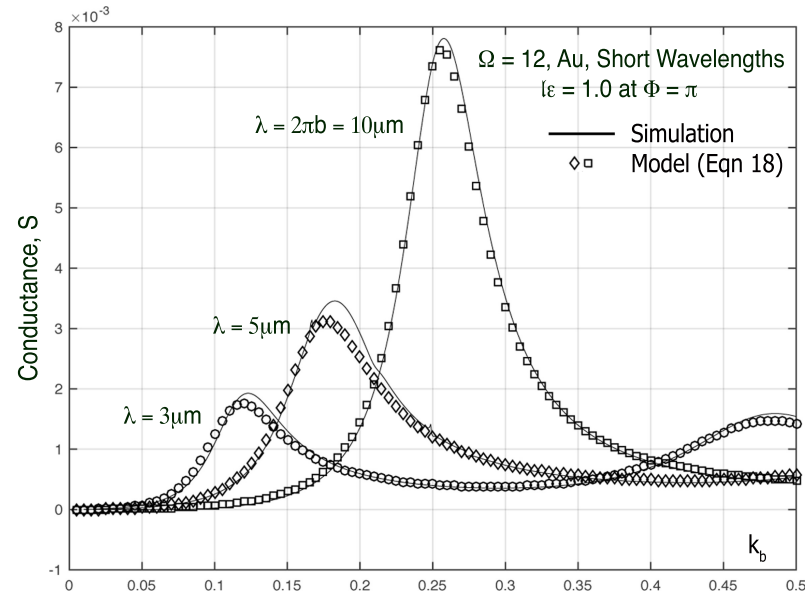

Fig. 6. Capacitive reactive load where $l_{\epsilon}=1.0$ is placed at $\phi=\pi$ in three differently sized nanoloops. Here $b=\{10 \mu \mathrm{m} / 2 \pi, 5 \mu \mathrm{m} / 2 \pi, 3 \mu \mathrm{m} / 2 \pi\}$. Simulation results are replicated well by the model in (18). See notes in the Appendix for simulation settings.

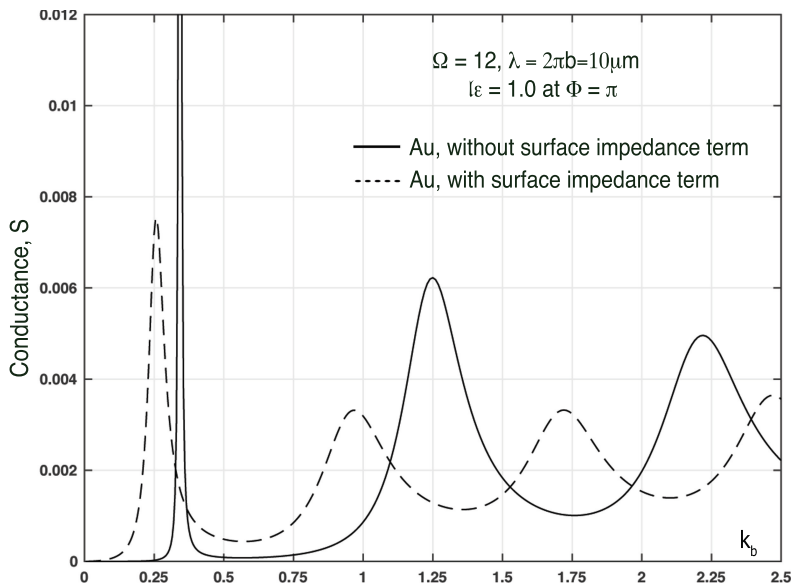

Fig. 7. Comparison of the conductances given by (18) of the $10-\mu \mathrm{m}$ gold loop in Fig. 6 with and without the use of the surface impedance term. The extra impedance causes higher losses and therefore smaller $\mathrm{Q}$ resonances and red shifting. value $l_{\epsilon}=1.0$ associated with a loop near its main resonance, $k_{b}=1.0$, has the reactance $-j X_{C}=-j \xi_{0}=-j 377 \Omega$ and a capacitive value of $C=\epsilon_{0} b l_{\epsilon}=8.85 b$ pf, where $b$ is in meters. In a similar way, inductive reactance is given by $j X_{L}=j \xi_{0} k_{b} l_{\mu}$, inductance $L=\mu_{0} b l_{\mu}$, and resistance by $R=\xi_{0} k_{b} r$, where $l_{\mu}$ and $r$ are the appropriate constants necessary to make the inductance and resistance desired at the given $k_{b}$. Fig. 6 shows the effects of using a capacitance of value $l_{\epsilon}=1.0$ in gold nanoloops with radii $b=\{10 \mu \mathrm{m} / 2 \pi$, $5 \mu \mathrm{m} / 2 \pi, 3 \mu \mathrm{m} / 2 \pi\}$. A resonance appears in the region below $k_{b}=0.5$, the "subwavelength" region. The surface impedance of the gold causes wavelength scaling and is the ultimate cause of the $k_{b}$ compression that appears in the figure (that is, the movement of the peak toward the left, a red shift scaling). This is a resonance saturation explained in [4].

It is the addition of the surface impedance term to legacy theory that allows for the extension to higher frequencies. Fig. 7 compares the conductances of the $\lambda=2 \pi$ $b=10-\mu \mathrm{m}$ gold loop shown in Fig. 6 when the term is used

in (18) and when it is not. When the term is not used, there is nothing in the legacy theory to distinguish the gold loop from a PEC loop; the conductance, therefore is the same as it would be at low frequencies for a PEC loop; a very high$\mathrm{Q}$ sub-wavelength resonance occurs near $k_{b}=0.35$ with the main resonance near $k_{b}=1.25$. With the term, using data for gold, the subwavelength resonance still exists, but its $Q$ is much smaller and all of the resonances have red shifted. The subwavelength resonance red shifts to $k_{b}=0.25$. Indeed, use of the surface impedance term is vital for reproducing actual behavior.

\section{CONCLUSION}

The original early work on impedance loaded loop antennas has been extended to the high $\mathrm{GHz}, \mathrm{THz}$, and optical regions for thin-wire, PEC, lossy, and frequency-dependent metal rings. The examples given in the early work using resistive loads was confirmed using a modern simulation tool, as was the extended theory using capacitive loads. The principal additions to the theory of loop antennas are twofold: 1) the inclusion of a surface impedance in the main legacy theory to allow for extension to the optical frequencies and 2) the clarification of the multiple gap mathematics using Einstein notation, which now also includes the surface impedance. The Appendix contains a derivation of the inductance, capacitance, and resistance of any thin-wire closed loop made of noble metals at any wavelength. MATLAB code is available from the author upon request.

\section{APPENDIX}

\section{A. RLC Element Derivation for Closed Loops}

The input impedance of a closed loop of a loop or nanoring at any frequency may be calculated in the following way. From (1), with $\phi=0$

$$
\sum_{m=0}^{\infty}\left[\frac{1}{Z_{m}^{\prime}}\right]=\left[\frac{1}{Z_{0}^{\prime}}+\sum_{1}^{\infty} \frac{1}{Z_{m}^{\prime}}\right] .
$$

Remembering that the impedance is complex

$$
\begin{aligned}
& Z^{*}=\frac{Z^{*} Z}{Z}=|Z|^{2}\left[\frac{1}{Z_{0}^{\prime}}+\sum_{1}^{\infty} \frac{1}{Z_{m}^{\prime}}\right] \\
&=|Z|^{2}\left[\frac{Z_{0}^{\prime *}}{Z_{0}^{\prime *} Z^{\prime} 0}+\sum_{1}^{\infty} \frac{Z_{m}^{\prime *}}{Z_{m}^{\prime *} Z_{m}^{\prime}}\right] \\
&=|Z|^{2}\left[\frac{R_{0}^{\prime}-i X_{0}^{\prime}}{\left|Z_{0}^{\prime}\right|^{2}}+\sum_{1}^{\infty} \frac{R_{m}^{\prime}-i X_{m}^{\prime}}{\left|Z_{m}^{\prime}\right|^{2}}\right] \\
&=|Z|^{2}\left[\left(\frac{R_{0}^{\prime}}{\left|Z_{0}^{\prime}\right|^{2}}+\sum_{1}^{\infty} \frac{R_{m}^{\prime}}{\left|Z_{m}^{\prime}\right|^{2}}\right)\right. \\
&\left.\quad-i\left(\frac{X_{0}^{\prime}}{\left|Z_{0}^{\prime}\right|^{2}}+\sum_{1}^{\infty} \frac{X_{m}^{\prime}}{\left|Z_{m}^{\prime}\right|^{2}}\right)\right] \\
&=R-i X .
\end{aligned}
$$


Equation (21) gives the total resistance and reactance of the loop, taking into account all of the modal impedances. It is, of course, just the complex conjugate of $Z$ which can be calculated from the definitions. The reactance in (21) can be expanded to give the total inductance and capacitance of the loop. Remembering that the capacitance has no zero mode

$$
\begin{aligned}
X & =|Z|^{2}\left[\frac{X_{0}^{\prime}}{\left|Z_{0}^{\prime}\right|^{2}}+\sum_{1}^{\infty} \frac{X_{m}^{\prime}}{\left|Z_{m}^{\prime}\right|^{2}}\right] \\
X_{L} & =\omega L=|Z|^{2}\left[\frac{\omega L_{0}^{\prime}}{\left|Z_{0}^{\prime}\right|^{2}}+\sum_{1}^{\infty} \frac{\omega L_{m}^{\prime}}{\left|Z_{m}^{\prime}\right|^{2}}\right] \\
X_{C} & =\frac{1}{\omega C}=|Z|^{2} \sum_{1}^{\infty} \frac{1 /\left(\omega C_{m}^{\prime}\right)}{\left|Z_{m}^{\prime}\right|^{2}} .
\end{aligned}
$$

Reducing, we have for $L$ and $C$

$$
\begin{aligned}
& L=\mu_{0} b l_{\mu}=|Z|^{2}\left[\frac{L_{0}^{\prime}}{\left|Z_{0}^{\prime}\right|^{2}}+\sum_{1}^{\infty} \frac{L_{m}^{\prime}}{\left|Z_{m}^{\prime}\right|^{2}}\right] \\
& \frac{1}{C}=\frac{1}{\epsilon_{0} b l_{\epsilon}}=|Z|^{2} \sum_{1}^{\infty} \frac{1 / C_{m}^{\prime}}{\left|Z_{m}^{\prime}\right|^{2}}
\end{aligned}
$$

These are functions of $k_{b}$. The prime, of course, refers to the elemental values when the surface impedance is taken into account.

\section{B. Simulation Methodology}

MWS by $\mathrm{CST}^{2}$ was used to produce the results appearing in Figs. 5 and 6. The low-frequency simulations for the first of Figs. 5 and 6 use an $\Omega=10$, PEC torus with middle circumference, $2 \pi b=3 \mathrm{~m}$ (about $100 \mathrm{MHz}$ ). A discrete port with an internal resistance, $R_{\text {port }}$, is placed across a gap of width $0.05 b$ at $\phi=0$. The resistive loads are established as lumped elements across a similar gap at $\phi=\pi$. The schematic is not used.

The high-frequency simulations in the second of Figs. 5 and 6 use an $\Omega=12$ gold torus of various sizes as noted in the text. A port resistance and two gaps are used as for the previous figure. The permittivity of the gold follows the permittivity model described in great detail in [4].

Each gap acts as a capacitive reactance, $X_{g}$. The gap reactance at $\phi=0$ affects the input impedance, $Z_{i n}$, for some ranges of $k_{b} ; Z_{i n}$ is what MWS reports, and therefore, for a proper comparison with the results of the model, based on (18), $X_{g}$ must be removed (see Fig. 8). This can be done by assuming that it is a flat plate capacitance in parallel across the input loop impedance. On the other hand, the gap reactance at $\phi=\pi$ does not have to be removed because the load resistance, $R_{L}$, is much smaller than the gap reactance; moreover, it has very little effect on the resonances.

Maximum power transfer into the loop occurs when the port resistance matches $Z_{\text {in }}$. The proper $R_{\text {port }}$ to use is discovered

\footnotetext{
${ }^{2}$ Computer Simulation Technology AG, MWS, 2012, Darmstadt, Germany. www.cst.com
}

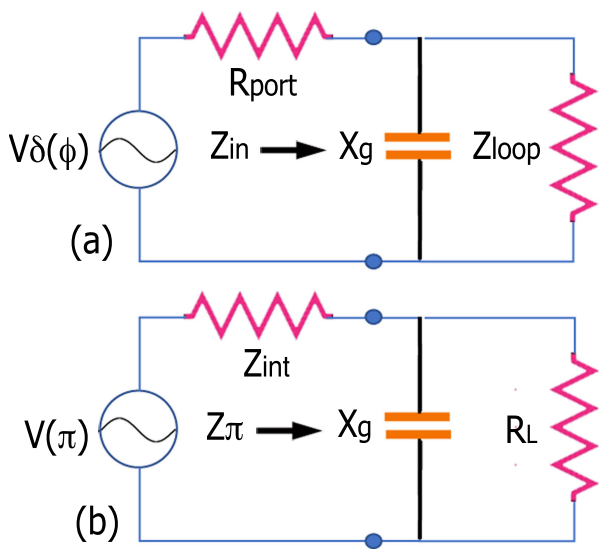

Fig. 8. Equivalent circuits representations for the loop at (a) $\phi=0$ and (b) $\phi=\pi$. MWS reports $Z_{i n}$ and therefore $X_{g}$ needs to be removed from the results for a fair comparison with the model. This is not true for $X_{g}$ at $\phi=\pi$ (see the text).

iteratively. A first solution using $R=50 \mathrm{ohm}$ is tried; the real part of the resulting input loop impedance at the main resonance (near $k_{b}=1.0$ where the imaginary part is zero) is then used for the port resistance in the next trial run. This continues until convergence, often after just two trials. The capacitance of the gap is not removed before the loop resistance is identified. The internal port impedance has no bearing on the comparison of simulation and model results.

The discrete port supplied in the MWS simulator does not make good contact with the wire. This is particularly true when the material of the torus is something other than PEC. Consequently, small PEC spheres are placed inside the torus on either side of the gaps, but protruding a bit into the gap so to provide a connection point for the port. This seems to provide adequate contact for the solver.

The theoretical model was derived for vanishingly thin loops. The thickness of the loop adds additional inductance that the simulations naturally take into account. For a fair comparison with the model, that inductance needs to be added in the model. This can be done effectively by introducing an inductance, approximate to first order, in series with the loop at the input. In other words, add an input impedance, $Z_{1}^{1}=\xi_{0} k_{b}\left(r+j l_{\mu}\right)$, in series with $V_{1}$ in (12). The resistance added to the loops used in Fig. 5 was $R=\xi_{0} k_{b} r$ where $r=$ 0.025 and the inductance was $L=\mu_{0} b l_{\mu}$ where $l_{\mu}=0.110$. The thickness of the $\Omega=12$ loops in Fig. 6 adds no appreciable resistance or inductance at the very high frequencies studied.

MATLAB code, written to reproduce (11) and (12) for any number of loads around the loop, is available from the author upon request.

\section{ACKNOWLEDGMENT}

The author would like to thank Dr. K. R. Catchpole and Dr. T. P. White, both from the Research School of Engineering, The Centre for Sustainable Energy Systems, The Australian National University, Canberra, Australia, and Dr. D. Roy Chowdhury from Mahindra École Centrale, Hyderabad, India, for their help on initial drafts of this paper. 
They would also like to thank the reviewers for excellent and fruitful suggestions.

\section{REFERENCES}

[1] J. E. Storer, "Impedance of thin-wire loop antennas," Trans. Amer. Inst. Elect. Eng., vol. 75, pp. 606-619, Nov. 1956.

[2] T. T. Wu, "Theory of the thin circular loop antenna," J. Math. Phys., vol. 3, no. 6, pp. 1301-1304, 1962.

[3] K. Iizuka, "The circular loop antenna multiloaded with positive and negative resistors," IEEE Trans. Antennas Propag., vol. 13, no. 1, pp. 7-20, Jan. 1965.

[4] A. F. McKinley, T. P. White, and K. R. Catchpole, "Theory of the circular closed loop antenna in the terahertz, infrared, and optical regions," J. Appl. Phys., vol. 114, no. 4, p. 044317, 2013. [Online]. Available: http://scitation.aip.org/content/aip/journal/jap/114/4/10.1063/1.4816619

[5] J. A. Stratton, Electromagnetic Theory. New York, NY, USA: McGraw-Hill, 1941.

[6] L. Novotny, "Effective wavelength scaling for optical antennas," Phys. Rev. Lett., vol. 98, no. 26, p. 266802, Jun. 2007. [Online]. Available: http://link.aps.org/doi/10.1103/PhysRevLett.98.266802

[7] A. F. McKinley, T. P. White, I. S. Maksymov, and K. R. Catchpole, "The analytical basis for the resonances and anti-resonances of loop antennas and meta-material ring resonators," J. Appl. Phys., vol. 112, no. 9, p. 094911, 2012. [Online]. Available: http://link.aip.org/link/? JAP/112/094911/1

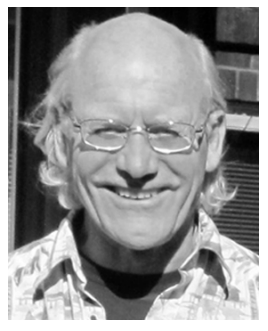

Arnold F. McKinley received the master's degrees in engineering-economic systems and electrical engineering from Stanford University, Stanford, CA, USA, in 1973 and 1974, respectively, the master's degree in comparative religions from Graduate Theological Union, Berkeley, CA, USA, in 1989, and the Ph.D. degree from The Australian National University, Canberra, ACT, Australia, in 2014, with a focus on the resonances of nanorings for the use as antennas on solar cells.

He was with the Institute for Energy Studies, Stanford, and with the Center for the Study of Social Policy at the Stanford Research Institute (now SRI International), Menlo Park, CA, USA, in the 1970s. In the early 1980s, he taught at the departments of Physics and Electrical Engineering, San Diego State University, San Diego, CA, USA For the next 25 years, he was a Professional Programmer on contract and internally for various scientific laboratories and institutions, including Apple Computer, X-Rite Technology, the Minolta Laboratory Systems, and the Center for Research in Mathematics and Science Education. In 1995, he founded MetaMind Software to develop educational software for engineering and science students. He is currently a Teaching Fellow at the Department of Electronics and Electrical Engineering, University College London, London, U.K., where he coordinates the Renewable Energy Minor Program for the Faculty of Engineering, focuses on issues concerned with the integration of renewables on the electrical grid in the U.S., Australia, and Europe, and continues research on nanoring antennas for THz and optical solutions. 


\title{
Theory of Impedance Loaded Loop Antennas and Nanorings From RF to Optical Wavelengths
}

\author{
Arnold F. McKinley
}

\begin{abstract}
The analytical theory of perfectly conducting thinwire closed-loop antennas with multiple loads in the periphery was formally derived in the 1950s and 1960s. In this paper, it is extended to loop antennas and nanorings for use in communications, in the "Internet of things," and as metamaterials. The new derivation relies on recent work from 2013 that incorporates the surface impedance of metal wires into the standard theory, thus pushing its applicability into the gigahertz, terahertz, and optical regimes. Surface impedance effects cause losses and phase shifts in the current within the loop, which in turn cause wavelength scaling and degradation of signal strength. These effects are modeled using a critical point transition model of permittivity and of the index of refraction. The new results therefore extend standard loop antenna theory so that it now includes characteristics of multiply loaded loops over a very broad spectrum from radio frequencies to the optical region. The new model is verified using modern simulation tools. The examples given here include resistive and capacitive loading.
\end{abstract}

Index Terms-Antenna theory, critical point model, Internet of things, loop antennas, metamaterials, multiply loaded antennas, nanorings, nanotechnology, wavelength scaling.

\section{INTRODUCTION}

$\mathbf{S}$ TANDARD loop antenna theory appeared in the $1950 \mathrm{~s}$ and 1960s with the work in Storer [1] and Wu [2]. Solutions of the differential equations were difficult, even with the assumption of closed ring vanishingly thin perfectly conducting (PEC) wires. Later, lizuka [3] broke the need for a closed ring, enabling the theory to include multiple loads evenly spaced around the periphery. It was not until 2013 [4] that the surface impedance effects, which cause loss and phase shift, were incorporated into the closed-loop theory, using a critical point transition model of permittivity and of the index of refraction. This enhancement brought the standard closed ring theory to the gigahertz $(\mathrm{GHz})$, terahertz $(\mathrm{THz})$, and optical regions.

In this paper, the surface impedance effects are coupled with the multiply loaded ring theory so that the entire theory becomes available to researchers working in $\mathrm{GHz}$ and $\mathrm{THz}$ communication and in $\mathrm{GHz}, \mathrm{THz}$, and optical metamaterials.

In Section II, the original cumbersome matrix notation used to derive the theory of multiply loaded loops is replaced with

Manuscript received July 20, 2016; revised February 11, 2017; accepted March 8, 2017.

The author is with the Department of Electronic and Electrical Engineering, University College London, London WC1E 7JE, U.K. (e-mail: arni.mckinley@ucl.ac.uk).

Color versions of one or more of the figures in this paper are available online at http://ieeexplore.ieee.org.

Digital Object Identifier 10.1109/TAP.2017.2682219

the easier Einstein notation. From the very first step, the derivation includes the characteristics of metals at high frequencies. The resulting new theory yields proper wavelength scaling, an important characterization of metals operating at very high frequency [5, Sec. 9.17] [6]. Two examples are given, one with resistive loads and one with capacitive loads. Conclusions follow with the Appendix giving a heretofore unreported derivation of the complete resistance, inductance, and capacitance of loop antennas, which, when new theory is included, yields these values for the entire spectrum, RF through optical. The second part of the Appendix gives proper simulation settings for verification of the new theory using CST's Microwave Studio (MWS) ${ }^{1}$ tool.

Two variables are important to understand. The first is the parameter $k_{b}=2 \pi b / \lambda$, where $\lambda$ is the incident or driving wavelength. Frequency increases as $k_{b}$ increases. The second is the thickness measure $\Omega=2 \ln (2 \pi b / a)$. Larger values of $\Omega$ refer to larger aspect ratios, $b / a$, and therefore to thinner wires. Loop antennas and nanorings where $\Omega \geq 10$ are said to follow the "thin-wire" approximation, for which all of the derivations here apply.

\section{Modern Derivation of Multiply LoAded Loops}

\section{A. Current Distribution}

The principle theory of closed thin-wire loop antennas, given in [1] and [2], was extended to $\mathrm{GHz}, \mathrm{THz}$, and optical frequencies by [7] and [4]. In summary, a delta-function voltage generator, $V_{0} \delta(\phi)$, across an infinitesimal gap at $\phi=0$ provides a broad spectrum source for the loop (see Fig. 1). The current distribution for a completely closed ring without load is given by

$$
\begin{aligned}
I(\phi) & =V_{0} \sum_{m=-\infty}^{\infty}\left[\frac{e^{i m \phi}}{Z_{m}^{\prime}}\right] \\
\text { where } Z_{M}^{\prime} & =j \pi \xi_{0} a_{m}+(b / a) Z_{s} .
\end{aligned}
$$

The standard theory uses only the single term $Z_{m}=$ $j \pi \xi_{0} a_{m}$. The addition of $(b / a) Z_{s}$ extends the theory to the higher frequency ranges. $Z_{s}$ is the surface impedance of the metal material of the wire in all frequency regimes. A detailed definition and discussion of this impedance is given in [4].

The input impedance $Z_{\mathrm{CL}}$ and the input admittance $Y_{\mathrm{CL}}$ of the closed loop are given by placing the driving input voltage

\footnotetext{
${ }^{1}$ Computer Simulation Technology AG, MWS. 2012. Darmstadt, Germany. www.cst.com.
} 


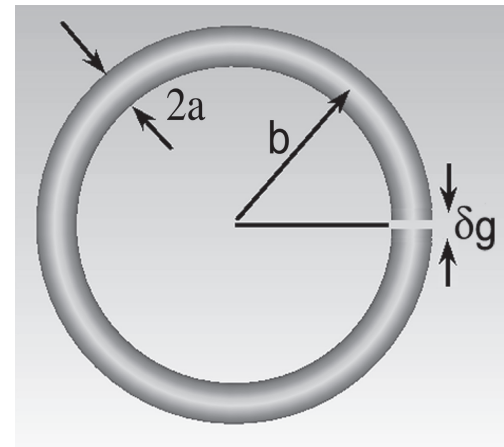

Fig. 1. Loop geometry. The gap is infinitesimally small and across it is placed a delta-function voltage generator to provide a broad spectrum source.

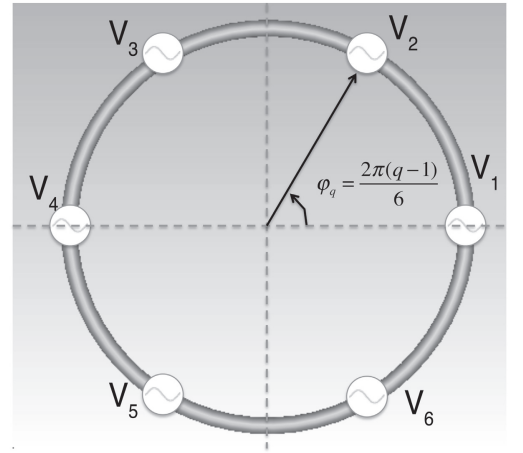

Fig. 2. Loop geometry of multiple sources and loads. The voltage sources marked " $V_{q}$ " are evenly spaced around the ring. The notation is short for " $V_{q} \delta\left(\phi-\phi_{q}\right)$ ".

at $\phi=0$

$$
Z_{\mathrm{CL}}=\frac{V_{0}}{I(0)}=\left[\sum_{m=-\infty}^{\infty} \frac{1}{Z_{m}^{\prime}}\right]^{-1}=\frac{1}{Y_{\mathrm{CL}}} .
$$

Iizuka [3] extended the low-frequency theory to include loads placed in the periphery of the loop. Unfortunately, the notation used in his paper is cumbersome and the more useful Einstein notation clarifies his approach and makes calculations easier. The derivation for loops with load impedances begins by first placing a number of $V_{q} \delta(\phi)$ voltage sources evenly around the ring separated by an angle $\Delta \phi=2 \pi / M$ where $M$ is the number of impedances, as shown in Fig. 2. Suppose $M=6$; the sources are then at the angles $\phi_{q}=2 \pi(q-$ 1) $/ 6, q=\{1, \ldots, 6\}$. The current in the ring results from a superposition of the currents due to all of the voltage sources, $V_{q}$. Each current has the form given by (1) and therefore is a distributed function of the angle, $\phi$. The total current is

$$
\begin{aligned}
I(\phi) & =\sum_{q=1}^{M} I_{q}(\phi) \\
& =\sum_{q=1}^{M}\left(\sum_{m=-\infty}^{\infty} \frac{e^{i m(\phi-2 \pi(q-1) / M)}}{Z_{m}^{\prime}}\right) V_{q} \\
& \equiv \sum_{q=1}^{M} Y(\phi, q) V_{q} .
\end{aligned}
$$

Since each $Z_{m}^{\prime}$ is given by (1), the surface impedance of 101 the wire is automatically included and Iizuka's theory is automatically extended to the higher frequency range.

Note that at the source $p$

$$
\begin{aligned}
Y\left(\phi_{p}, q\right) & =\sum_{m=-\infty}^{\infty} \frac{e^{i m(2 \pi(p-q) / M)}}{Z_{m}^{\prime}} \\
& \equiv\left[Y_{p q}\right] .
\end{aligned}
$$

This lends itself to matrix notation. The term $\left[Y_{p q}\right]$ is a square matrix with $M \times M$ elements; $p$ is the row counter and $q$ the column counter. $Y(\phi, q)$ in (3) becomes a vector, $Y_{q}(\phi)$. Then (3) becomes

$$
\begin{aligned}
I(\phi) & =\sum_{q=1}^{M}\left[Y_{q}(\phi)\right]\left[V_{q}\right] \\
& =Y^{q}(\phi) V_{q}
\end{aligned}
$$

where the last line uses the Einstein summation rule (repeated indices are summed over); the upper index refers to the elements in a horizontal vector and the subscript counter refers to the elements in a vertical vector. Similarly

$$
\left[Y_{p q}\right] \rightarrow Y_{q}^{p} .
$$

In order to include impedances in the formalism, series impedances (voltage sinks) are added to the voltage sources. $V_{q}$ transforms as

$$
V_{q} \rightarrow V_{q}-Z_{q}^{k} I_{k} .
$$

Setting $V_{q}$ to 0 eliminates the generator leaving only the impedance and vice versa. The current $I_{k}$ is the current at the source (with its distinct counter to avoid confusion), that is, $I_{k}$ means $I\left(\phi_{k}\right)$. $Z_{q}^{k}$ is a square $M \times M$ diagonal matrix in which the diagonal terms are the impedances at each of the voltage sources. This approach provides enough information to find the current at each impedance node. Setting $\phi_{p}$ in (5) and using (6)

$$
\begin{aligned}
I_{p} & =Y_{p}^{q} V_{q} \rightarrow Y_{p}^{q}\left(V_{q}-Z_{q}^{k} I_{k}\right) \\
& =Y_{p}^{q} V_{q}-Y_{p}^{q} Z_{q}^{k} I_{k} \\
I_{p}+Y_{p}^{q} Z_{q}^{k} I_{k} & =Y_{p}^{q} V_{q} \\
\left(\mathcal{I}_{p}^{k}+Y_{p}^{q} Z_{q}^{k}\right) I_{k} & =Y_{p}^{q} V_{q} .
\end{aligned}
$$

The term $\mathcal{I}_{p}^{k}$ is the diagonal identity matrix. To simplify the notation, define

$$
f_{p}^{k} \equiv \mathcal{I}_{p}^{k}+Y_{p}^{q} Z_{q}^{k} .
$$

Equation (8) becomes

$$
I_{k}=\left[f^{-1}\right]_{k}^{p} Y_{p}^{q} V_{q}
$$

where the inverse of the matrix $f_{p}^{k}$ has been taken. When (7) and (10) are substituted into the sum (5), the current at any angle in the ring results

$$
\begin{aligned}
I(\phi) & =Y^{q}(\phi)\left(V_{q}-Z_{q}^{k} I_{k}\right) \\
& =Y^{q}(\phi) V_{q}-Y^{q}(\phi) Z_{q}^{k} I_{k} \\
& =Y^{j}(\phi) V_{j}-Y^{q}(\phi) Z_{q}^{k}\left[f^{-1}\right]_{k}^{p} Y_{p}^{j} V_{j} .
\end{aligned}
$$




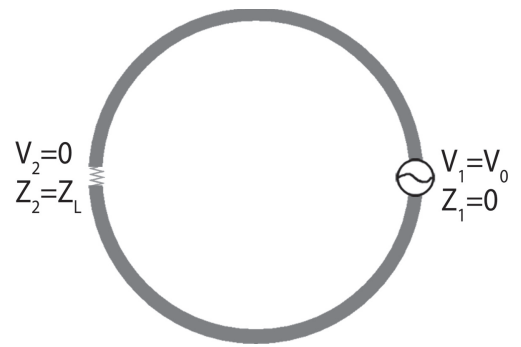

Fig. 3. Ring with one driving source and a load at $\phi=\pi$ to show a formal solution using (11).

The last line requires a change in the counter notation to prevent counter confusion. Notice the difference between (5) and (11). Each voltage generator $V_{q}$ in the former has been extended to include a load impedance.

\section{B. Input Impedance}

The driving point impedance and admittance occur when there is only one driving source. In this case, the source can be at any of the $\mathrm{M}$ angles spread evenly around the ring. Taking it at $\phi=0$, the impedance and admittance are calculated by dividing the source voltage, $V_{1}=V$, by the current at $\phi=0$

$$
Z_{\text {in }} \equiv \frac{V}{I(0)}=\left[Y^{1}(0)-Y^{q}(0) Z_{q}^{k}\left[f^{-1}\right]_{k}^{p} Y_{p}^{1}\right]^{-1} \text {. }
$$

\section{A. Resistive Loads}

As examples, let us explore singly loaded resistive and capacitive loops. In the first case, place in a PEC loop, a load impedance at $\phi_{2}=\pi$, and the drive source at $\phi_{1}=0$, as in Fig. 3. Then, $M=2, V_{1}=V$ volt, $V_{2}=0 ; Z_{2}^{2} \equiv Z_{L}$, while all other impedance elements are zero. The matrix elements, $Y_{p}^{q}$, are calculated as follows:

$$
Y=\left[\begin{array}{cc}
Y_{\mathrm{CL}} & Y_{\pi} \\
Y_{\pi} & Y_{\mathrm{CL}}
\end{array}\right]
$$

where

$$
Y_{\mathrm{CL}} \equiv \sum_{m=-\infty}^{\infty} \frac{1}{Z_{m}^{\prime}} \text { and } Y_{\pi} \equiv \sum_{m=-\infty}^{\infty} \frac{(-1)^{m}}{Z_{m}^{\prime}} .
$$

The matrix elements for $f$ are

$$
f=\left[\begin{array}{cc}
1 & Y_{\pi} Z_{L} \\
0 & 1+Y_{\mathrm{CL}} Z_{L}
\end{array}\right]
$$

and the current in (10) is

$$
\begin{aligned}
& I_{1}=\left[\frac{Y_{\mathrm{CL}}+Z_{L}\left(Y_{\mathrm{CL}} Y_{\mathrm{CL}}-Y_{\pi} Y_{\pi}\right)}{1+Y_{\mathrm{CL}} Z_{L}}\right] V \\
& I_{2}=\left[\frac{Y_{\pi}}{1+Y_{\mathrm{CL}} Z_{L}}\right] V .
\end{aligned}
$$

The total current is given by expanding (11)

$$
\left.I(\phi)=V\left[Y^{1}(\phi)-\frac{Y_{\pi} Z_{L}}{1+Y_{\mathrm{CL}} Z_{L}} Y^{2}(\phi)\right)\right] .
$$

These results reproduce Iizuka's equations (18), (19), and (22). The input impedance for the example can be found from (16)

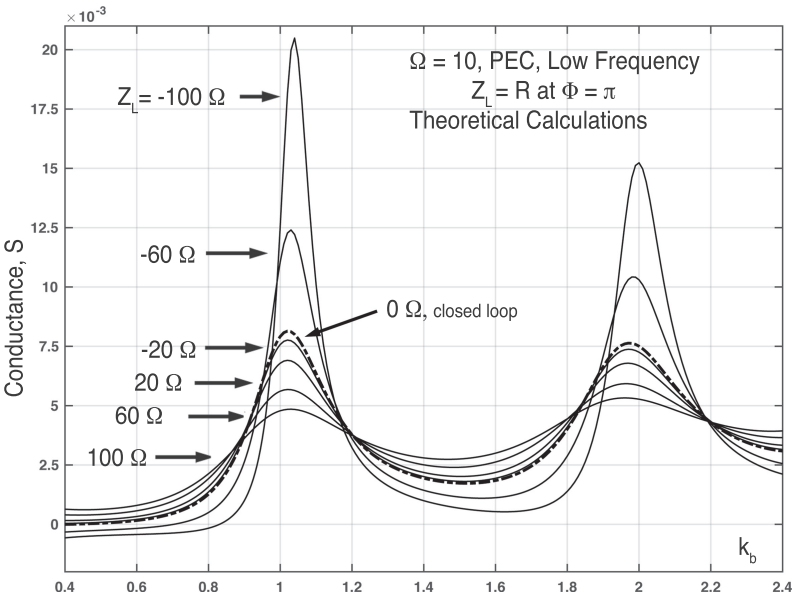

Fig. 4. Input admittance of an $\Omega=10$ and $2 \pi b=30$-m PEC loop with various resistive loads at $\phi=\pi$ using (18).

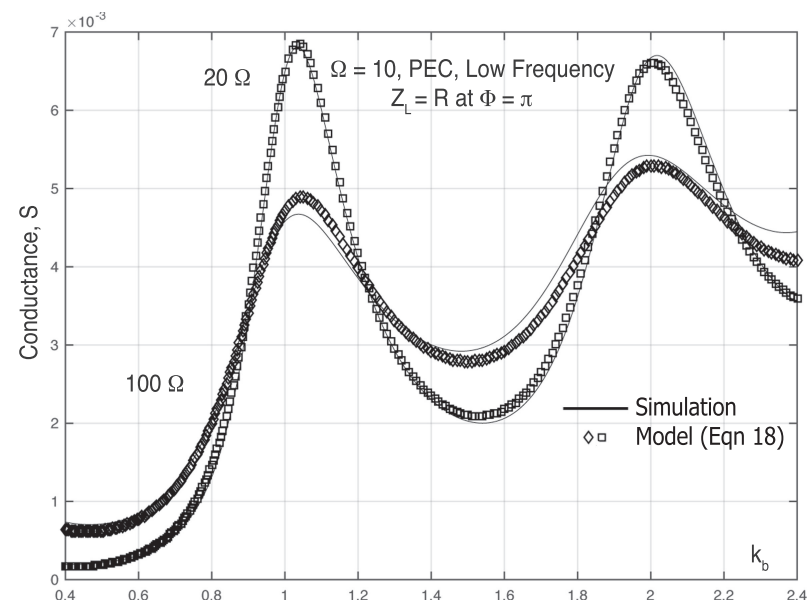

Fig. 5. 20- and 100- $\Omega$ curves of Fig. 4 compared with those given by simulation. See notes in the Appendix for simulation settings.

since $I_{1} \equiv I(0)$, or from (17) setting $\phi=0$

$$
Z_{\text {in }}=\frac{V}{I(0)}=\frac{1+Y_{\mathrm{CL}} Z_{L}}{Y_{\mathrm{CL}}+Z_{L}\left(Y_{\mathrm{CL}} Y_{\mathrm{CL}}-Y_{\pi} Y_{\pi}\right)} .
$$

The conductance of six different loop antennas where $Z_{L}=R=\{-100,-60,-20,20,60,100\}$, shown in Fig. 4 duplicates [3, Fig. 8]. See [3] for other resistive examples. The negative resistances were fabricated in [3] using Esaki diodes. Note that negative resistance can generate high resonances. Fig. 5 checks the validity of the model by comparing the 20 - and $100-\Omega$ curves of Fig. 4 with curves obtained by modern simulation. The correspondence is strong.

\section{B. Capacitive Loads}

As another example of the application of (18), set $Z_{L}$ to a capacitive reactive load. Using $\omega=k_{b} c / b$, where $c$ is the speed of light, the load may be expanded

$$
Z_{L}=Z_{\beta}=-j \frac{1}{\omega C}=-j \frac{\xi_{0}}{k_{b} l_{\epsilon}} .
$$

$k_{b}$ normalizes the reactance to the size of the loop and $l_{\epsilon}$ becomes a measure of capacitance. For example, a capacitor of 


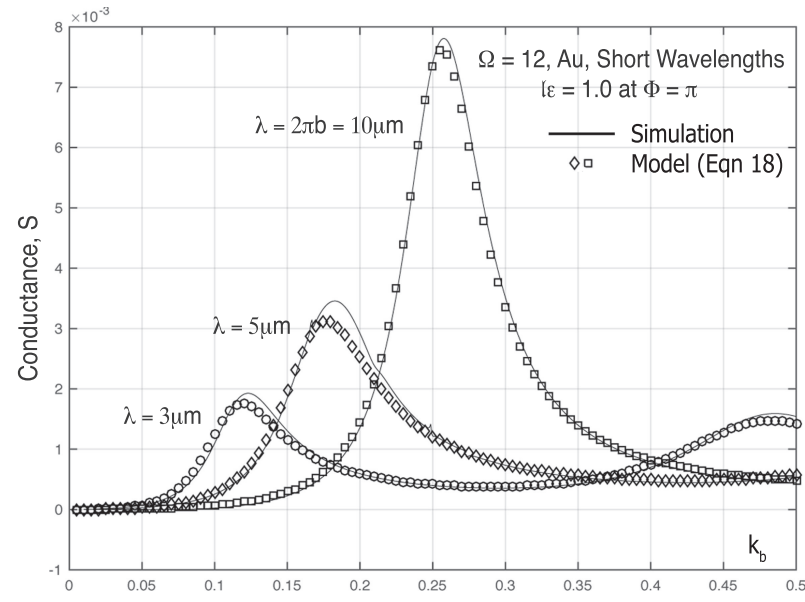

Fig. 6. Capacitive reactive load where $l_{\epsilon}=1.0$ is placed at $\phi=\pi$ in three differently sized nanoloops. Here $b=\{10 \mu \mathrm{m} / 2 \pi, 5 \mu \mathrm{m} / 2 \pi, 3 \mu \mathrm{m} / 2 \pi\}$. Simulation results are replicated well by the model in (18). See notes in the Appendix for simulation settings.

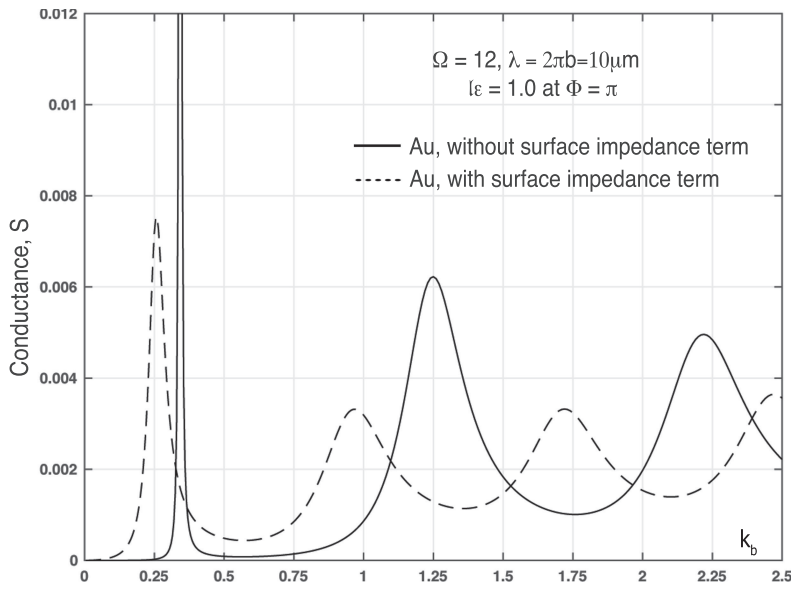

Fig. 7. Comparison of the conductances given by (18) of the $10-\mu \mathrm{m}$ gold loop in Fig. 6 with and without the use of the surface impedance term. The extra impedance causes higher losses and therefore smaller $\mathrm{Q}$ resonances and red shifting. value $l_{\epsilon}=1.0$ associated with a loop near its main resonance, $k_{b}=1.0$, has the reactance $-j X_{C}=-j \xi_{0}=-j 377 \Omega$ and a capacitive value of $C=\epsilon_{0} b l_{\epsilon}=8.85 b$ pf, where $b$ is in meters. In a similar way, inductive reactance is given by $j X_{L}=j \xi_{0} k_{b} l_{\mu}$, inductance $L=\mu_{0} b l_{\mu}$, and resistance by $R=\xi_{0} k_{b} r$, where $l_{\mu}$ and $r$ are the appropriate constants necessary to make the inductance and resistance desired at the given $k_{b}$. Fig. 6 shows the effects of using a capacitance of value $l_{\epsilon}=1.0$ in gold nanoloops with radii $b=\{10 \mu \mathrm{m} / 2 \pi$, $5 \mu \mathrm{m} / 2 \pi, 3 \mu \mathrm{m} / 2 \pi\}$. A resonance appears in the region below $k_{b}=0.5$, the "subwavelength" region. The surface impedance of the gold causes wavelength scaling and is the ultimate cause of the $k_{b}$ compression that appears in the figure (that is, the movement of the peak toward the left, a red shift scaling). This is a resonance saturation explained in [4].

It is the addition of the surface impedance term to legacy theory that allows for the extension to higher frequencies. Fig. 7 compares the conductances of the $\lambda=2 \pi$ $b=10-\mu \mathrm{m}$ gold loop shown in Fig. 6 when the term is used

in (18) and when it is not. When the term is not used, there is nothing in the legacy theory to distinguish the gold loop from a PEC loop; the conductance, therefore is the same as it would be at low frequencies for a PEC loop; a very high$\mathrm{Q}$ sub-wavelength resonance occurs near $k_{b}=0.35$ with the main resonance near $k_{b}=1.25$. With the term, using data for gold, the subwavelength resonance still exists, but its $Q$ is much smaller and all of the resonances have red shifted. The subwavelength resonance red shifts to $k_{b}=0.25$. Indeed, use of the surface impedance term is vital for reproducing actual behavior.

\section{CONCLUSION}

The original early work on impedance loaded loop antennas has been extended to the high $\mathrm{GHz}, \mathrm{THz}$, and optical regions for thin-wire, PEC, lossy, and frequency-dependent metal rings. The examples given in the early work using resistive loads was confirmed using a modern simulation tool, as was the extended theory using capacitive loads. The principal additions to the theory of loop antennas are twofold: 1) the inclusion of a surface impedance in the main legacy theory to allow for extension to the optical frequencies and 2) the clarification of the multiple gap mathematics using Einstein notation, which now also includes the surface impedance. The Appendix contains a derivation of the inductance, capacitance, and resistance of any thin-wire closed loop made of noble metals at any wavelength. MATLAB code is available from the author upon request.

\section{APPENDIX}

\section{A. RLC Element Derivation for Closed Loops}

The input impedance of a closed loop of a loop or nanoring at any frequency may be calculated in the following way. From (1), with $\phi=0$

$$
\sum_{m=0}^{\infty}\left[\frac{1}{Z_{m}^{\prime}}\right]=\left[\frac{1}{Z_{0}^{\prime}}+\sum_{1}^{\infty} \frac{1}{Z_{m}^{\prime}}\right] .
$$

Remembering that the impedance is complex

$$
\begin{aligned}
& Z^{*}=\frac{Z^{*} Z}{Z}=|Z|^{2}\left[\frac{1}{Z_{0}^{\prime}}+\sum_{1}^{\infty} \frac{1}{Z_{m}^{\prime}}\right] \\
&=|Z|^{2}\left[\frac{Z_{0}^{\prime *}}{Z_{0}^{\prime *} Z^{\prime} 0}+\sum_{1}^{\infty} \frac{Z_{m}^{\prime *}}{Z_{m}^{\prime *} Z_{m}^{\prime}}\right] \\
&=|Z|^{2}\left[\frac{R_{0}^{\prime}-i X_{0}^{\prime}}{\left|Z_{0}^{\prime}\right|^{2}}+\sum_{1}^{\infty} \frac{R_{m}^{\prime}-i X_{m}^{\prime}}{\left|Z_{m}^{\prime}\right|^{2}}\right] \\
&=|Z|^{2}\left[\left(\frac{R_{0}^{\prime}}{\left|Z_{0}^{\prime}\right|^{2}}+\sum_{1}^{\infty} \frac{R_{m}^{\prime}}{\left|Z_{m}^{\prime}\right|^{2}}\right)\right. \\
&\left.\quad-i\left(\frac{X_{0}^{\prime}}{\left|Z_{0}^{\prime}\right|^{2}}+\sum_{1}^{\infty} \frac{X_{m}^{\prime}}{\left|Z_{m}^{\prime}\right|^{2}}\right)\right] \\
&=R-i X .
\end{aligned}
$$

212 213 214 215 216 217 218 219 220 221 
Equation (21) gives the total resistance and reactance of the loop, taking into account all of the modal impedances. It is, of course, just the complex conjugate of $Z$ which can be calculated from the definitions. The reactance in (21) can be expanded to give the total inductance and capacitance of the loop. Remembering that the capacitance has no zero mode

$$
\begin{aligned}
X & =|Z|^{2}\left[\frac{X_{0}^{\prime}}{\left|Z_{0}^{\prime}\right|^{2}}+\sum_{1}^{\infty} \frac{X_{m}^{\prime}}{\left|Z_{m}^{\prime}\right|^{2}}\right] \\
X_{L} & =\omega L=|Z|^{2}\left[\frac{\omega L_{0}^{\prime}}{\left|Z_{0}^{\prime}\right|^{2}}+\sum_{1}^{\infty} \frac{\omega L_{m}^{\prime}}{\left|Z_{m}^{\prime}\right|^{2}}\right] \\
X_{C} & =\frac{1}{\omega C}=|Z|^{2} \sum_{1}^{\infty} \frac{1 /\left(\omega C_{m}^{\prime}\right)}{\left|Z_{m}^{\prime}\right|^{2}} .
\end{aligned}
$$

Reducing, we have for $L$ and $C$

$$
\begin{aligned}
& L=\mu_{0} b l_{\mu}=|Z|^{2}\left[\frac{L_{0}^{\prime}}{\left|Z_{0}^{\prime}\right|^{2}}+\sum_{1}^{\infty} \frac{L_{m}^{\prime}}{\left|Z_{m}^{\prime}\right|^{2}}\right] \\
& \frac{1}{C}=\frac{1}{\epsilon_{0} b l_{\epsilon}}=|Z|^{2} \sum_{1}^{\infty} \frac{1 / C_{m}^{\prime}}{\left|Z_{m}^{\prime}\right|^{2}} .
\end{aligned}
$$

These are functions of $k_{b}$. The prime, of course, refers to the elemental values when the surface impedance is taken into account.

\section{B. Simulation Methodology}

MWS by $\mathrm{CST}^{2}$ was used to produce the results appearing in Figs. 5 and 6. The low-frequency simulations for the first of Figs. 5 and 6 use an $\Omega=10$, PEC torus with middle circumference, $2 \pi b=3 \mathrm{~m}$ (about $100 \mathrm{MHz}$ ). A discrete port with an internal resistance, $R_{\text {port }}$, is placed across a gap of width $0.05 b$ at $\phi=0$. The resistive loads are established as lumped elements across a similar gap at $\phi=\pi$. The schematic is not used.

The high-frequency simulations in the second of Figs. 5 and 6 use an $\Omega=12$ gold torus of various sizes as noted in the text. A port resistance and two gaps are used as for the previous figure. The permittivity of the gold follows the permittivity model described in great detail in [4].

Each gap acts as a capacitive reactance, $X_{g}$. The gap reactance at $\phi=0$ affects the input impedance, $Z_{i n}$, for some ranges of $k_{b} ; Z_{i n}$ is what MWS reports, and therefore, for a proper comparison with the results of the model, based on (18), $X_{g}$ must be removed (see Fig. 8). This can be done by assuming that it is a flat plate capacitance in parallel across the input loop impedance. On the other hand, the gap reactance at $\phi=\pi$ does not have to be removed because the load resistance, $R_{L}$, is much smaller than the gap reactance; moreover, it has very little effect on the resonances.

Maximum power transfer into the loop occurs when the port resistance matches $Z_{\text {in }}$. The proper $R_{\text {port }}$ to use is discovered

\footnotetext{
${ }^{2}$ Computer Simulation Technology AG, MWS, 2012, Darmstadt, Germany. www.cst.com.
}

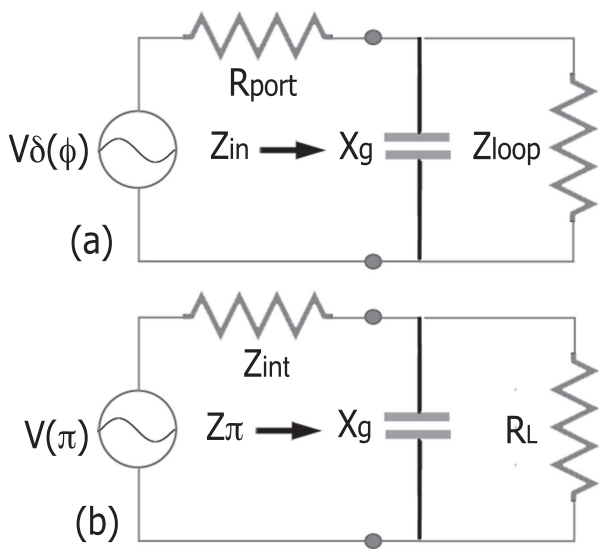

Fig. 8. Equivalent circuits representations for the loop at (a) $\phi=0$ and (b) $\phi=\pi$. MWS reports $Z_{i n}$ and therefore $X_{g}$ needs to be removed from the results for a fair comparison with the model. This is not true for $X_{g}$ at $\phi=\pi$ (see the text)

iteratively. A first solution using $R=50 \mathrm{ohm}$ is tried; the real part of the resulting input loop impedance at the main resonance (near $k_{b}=1.0$ where the imaginary part is zero) is then used for the port resistance in the next trial run. This continues until convergence, often after just two trials. The capacitance of the gap is not removed before the loop resistance is identified. The internal port impedance has no bearing on the comparison of simulation and model results.

The discrete port supplied in the MWS simulator does not make good contact with the wire. This is particularly true when the material of the torus is something other than PEC. Consequently, small PEC spheres are placed inside the torus on either side of the gaps, but protruding a bit into the gap so to provide a connection point for the port. This seems to provide adequate contact for the solver.

The theoretical model was derived for vanishingly thin loops. The thickness of the loop adds additional inductance that the simulations naturally take into account. For a fair comparison with the model, that inductance needs to be added in the model. This can be done effectively by introducing an inductance, approximate to first order, in series with the loop at the input. In other words, add an input impedance, $Z_{1}^{1}=\xi_{0} k_{b}\left(r+j l_{\mu}\right)$, in series with $V_{1}$ in (12). The resistance added to the loops used in Fig. 5 was $R=\xi_{0} k_{b} r$ where $r=$ 0.025 and the inductance was $L=\mu_{0} b l_{\mu}$ where $l_{\mu}=0.110$. The thickness of the $\Omega=12$ loops in Fig. 6 adds no appreciable resistance or inductance at the very high frequencies studied.

MATLAB code, written to reproduce (11) and (12) for any number of loads around the loop, is available from the author upon request.

\section{ACKNOWLEDGMENT}

The author would like to thank Dr. K. R. Catchpole and Dr. T. P. White, both from the Research School of Engineering, The Centre for Sustainable Energy Systems, The Australian National University, Canberra, Australia, and Dr. D. Roy Chowdhury from Mahindra École Centrale, Hyderabad, India, for their help on initial drafts of this paper. 
They would also like to thank the reviewers for excellent and fruitful suggestions.

\section{REFERENCES}

[1] J. E. Storer, "Impedance of thin-wire loop antennas," Trans. Amer. Inst. Elect. Eng., vol. 75, pp. 606-619, Nov. 1956.

[2] T. T. Wu, "Theory of the thin circular loop antenna," J. Math. Phys., vol. 3, no. 6, pp. 1301-1304, 1962.

[3] K. Iizuka, "The circular loop antenna multiloaded with positive and negative resistors," IEEE Trans. Antennas Propag., vol. 13, no. 1, pp. 7-20, Jan. 1965.

[4] A. F. McKinley, T. P. White, and K. R. Catchpole, "Theory of the circular closed loop antenna in the terahertz, infrared, and optical regions," J. Appl. Phys., vol. 114, no. 4, p. 044317, 2013. [Online]. Available: http://scitation.aip.org/content/aip/journal/jap/114/4/10.1063/1.4816619

[5] J. A. Stratton, Electromagnetic Theory. New York, NY, USA: McGraw-Hill, 1941.

[6] L. Novotny, "Effective wavelength scaling for optical antennas," Phys. Rev. Lett., vol. 98, no. 26, p. 266802, Jun. 2007. [Online]. Available: http://link.aps.org/doi/10.1103/PhysRevLett.98.266802

[7] A. F. McKinley, T. P. White, I. S. Maksymov, and K. R. Catchpole, "The analytical basis for the resonances and anti-resonances of loop antennas and meta-material ring resonators," J. Appl. Phys., vol. 112 no. 9, p. 094911, 2012. [Online]. Available: http://link.aip.org/link/? JAP/112/094911/1

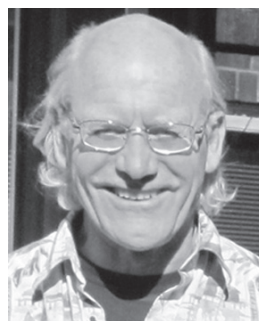

Arnold F. McKinley received the master's degrees in engineering-economic systems and electrical engineering from Stanford University, Stanford, CA, USA, in 1973 and 1974, respectively, the master's degree in comparative religions from Graduate Theological Union, Berkeley, CA, USA, in 1989, and the Ph.D. degree from The Australian National University, Canberra, ACT, Australia, in 2014, with a focus on the resonances of nanorings for the use as antennas on solar cells.

He was with the Institute for Energy Studies, Stanford, and with the Center for the Study of Social Policy at the Stanford Research Institute (now SRI International), Menlo Park, CA, USA, in the 1970s. In the early 1980s, he taught at the departments of Physics and Electrical Engineering, San Diego State University, San Diego, CA, USA For the next 25 years, he was a Professional Programmer on contract and internally for various scientific laboratories and institutions, including Apple Computer, X-Rite Technology, the Minolta Laboratory Systems, and the Center for Research in Mathematics and Science Education. In 1995, he founded MetaMind Software to develop educational software for engineering and science students. He is currently a Teaching Fellow at the Department of Electronics and Electrical Engineering, University College London, London, U.K., where he coordinates the Renewable Energy Minor Program for the Faculty of Engineering, focuses on issues concerned with the integration of renewables on the electrical grid in the U.S., Australia, and Europe, and continues research on nanoring antennas for THz and optical solutions. 